\title{
Motion Coordination for Multi-agent Networks
}

\author{
Francesco Bullo \\ Mechanical Engineering, \\ University of California at Santa Barbara, \\ http://motion.mee.ucsb.edu
}

\begin{abstract}
Motion coordination is an extraordinary phenomenon in biological systems, such as schools of fishes, as well as a remarkable tool for man-made groups of robotic vehicles and active sensors. Even though each individual agent has no global knowledge of the system, complex coordinated behaviors emerge from local interactions. In this talk I will describe some recently-developed models, algorithms and tools for motion coordination. Building on concepts from distributed computation, robotics and control theory, I investigate notions of robotic network, joint control and communication laws, and time complexity of coordination tasks. From an algorithmic viewpoint, the focus is on various coordination problems such as network deployment over a given region, rendezvous at a point, and vehicle routing. The proposed control and communication laws achieve the various coordination objectives requiring only spatially-distributed information.
\end{abstract}

